\title{
USING THE THEORY OF GROWING BODIES IN CALCULATION OF STRESS-STRAIN STATE OF THE PARTS MANUFACTURED APPLYING ADDITIVE CLADDING TECHNOLOGIES
}

\author{
I.K. SENCHENKOV ${ }^{1}$, I.A. RYABTSEV ${ }^{2}$, E. TURYK ${ }^{3}$ and O.P. CHERVINKO ${ }^{1}$ \\ ${ }^{1}$ S.P. Timoshenko Institute of Mechanics, NASU \\ 3 Nesterov Str., 03057, Kiev, Ukraine. E-mail: ang@imech.freenet.kiev.ua \\ ${ }^{2}$ E.O. Paton Electric Welding Institute, NASU \\ 11 Kazimir Malevich Str., 03680, Kiev, Ukraine. E-mail: office@paton.kiev.ua \\ ${ }^{3}$ Institute of Welding \\ 16-18 Bl. Czeslaw Str., 44-100, Gliwice, Poland. E-mail: eugeniusz.turyk@gliwice.pl
}

\begin{abstract}
Proceeding from the theory of growing bodies and unified model of flow, a model was developed for assessment of thermomechanical state of the part in multilayer cladding, as well as finite-element procedure of numerical realization of the model. In the case of cladding (building-up) a cylinder along its side surface it was established that the schematic of one-time building-up allows rather quickly obtaining qualitative evaluations of the level of stresses and strains at analysis of different variants of technological solutions for multilayer cladding of parts. However, it does not allow for the significant inhomogeneity and celullar structure of distributions, but just describes the smooth averaged change of characteristics of stresses and strains along the cylinder. It does not even allow studying such fine technological points as influence of different schematics of deposited bead overlapping on stress-strain state of the part. In this case, it is recommended to use the procedure of calculation by the schematic of bead-by-bead building-up (deposition). Generally satisfactory agreement of calculated and experimental data is indicative of the validity and reliability of the developed approach to modeling the thermomechanical processes in multilayer cladding of parts. 14 Ref., 9 Figures.
\end{abstract}

Keywords : cladding, additive technologies, theory of growing bodies, stress-strain state, residual stresses, deposited beads, deposited layers

By the generally accepted definition, additive technologies are technologies of manufacturing complex-shaped parts by 3D computer model by successive layer-by-layer deposition of material [1-4]. According to this definition, cladding can also be regarded as an additive technology. So far, however, at development of additive technologies with application of welding heat sources, attention was focused on laser and electron beam $[5,6]$. Less attention was paid to other methods, in particular cladding, as additive technologies. Not enough attention at analysis was also given to the fact that layer-by-layer deposition of molten metal in additive technologies leads to formation of a certain stress-strain state (SSS) in finished parts, which may result in residual deformations or crack initiation in them.

Experimental methods of SSS investigation are expensive and labour-consuming processes, providing quite limited information. Therefore, development of models and procedures of mathematical modeling of stress-strain and microstructural state of the parts during incrementing, residual state, as well as stresses during operation, is a highly urgent task.
The objective of this work is development of a model and procedure of calculation of SSS of parts, manufactured with application of additive cladding technologies. The procedure is based on the theory of growing bodies [7], which simulates multilayer cladding process to the greatest extent [8].

Mechanical behaviour of material is described by Bodner-Partom model [9], which includes the following relationships in rectangular Cartesian system of coordinates Oxyz:

- law of flow and equation of plastic incompressibility

$$
\dot{\varepsilon}_{i j}=\dot{\varepsilon}_{i j}^{\mathrm{p}}+\dot{\varepsilon}_{i j}^{\mathrm{e}}, \dot{\varepsilon}_{k k}^{\mathrm{p}}=0, \quad i, j=x, y, z ;
$$

- Prandtl-Reuss law of flow

$$
\dot{\varepsilon}_{i j}=\frac{D_{0}}{J_{2}^{1 / 2}} \exp \left[-\frac{1}{2}\left(\frac{K_{0}+K}{\sqrt{3 J_{2}}}\right)^{2 n}\right] s_{i j},
$$

where

$$
\begin{gathered}
J_{2}=\frac{1}{2} s_{i j} s_{i j} ; \quad s_{i j}=\sigma_{i j}-\frac{1}{3} \delta_{i j} \sigma_{k k} ; \\
\sigma_{k k}=\sigma_{x x}+\sigma_{y y}+\sigma_{z z} ;
\end{gathered}
$$


- evolution equation for isotropic strengthening parameter

$$
\dot{K}=m_{1}\left(K_{1}-K\right) \dot{W}_{p}, \quad K(0)=0,
$$

where $\dot{W}_{p}=\sigma_{i j} \dot{\varepsilon}_{i j}^{\mathrm{p}} ; D_{0}, K_{0}, K_{1}, m_{1}, n$ are the model parameters;

- Hooke's law

$$
\begin{gathered}
\sigma_{k k}=3 K_{V}\left(\varepsilon_{k k}-3 \alpha\left(\theta-\theta_{0}\right)\right), \\
S_{i j}=2 G\left(e_{i j}-\varepsilon_{i j}^{\mathrm{p}}\right), \quad e_{i j}=\varepsilon_{i j}-\frac{1}{3} \varepsilon_{k k} \delta_{i j},
\end{gathered}
$$

where $G, K_{v}, \alpha$ are the moduli of shear, volume compression and coefficient of linear thermal expansion.

Relationships are complemented by universal equations of quasi-static equilibrium and heat conductivity, as well as respective boundary and initial conditions.

Two models of the process of multilayer cladding of parts are considered. The first is bead-by-bead layer-by-layer cladding (Figure 1,a), the second is onetime layer-by-layer cladding (Figure 1, $b$ ).

The first incremental model allows for deposition of each individual bead in each of the layers. In the second model, called one-time cladding, theory of growing bodies is used for modeling successive onetime deposition of each individual layer. The time interval between joining these beads or layers, their dimensions, material temperature, cooling time, etc. are selected from the condition of their equivalence to geometrical, energy and other parameters of technological process of cladding.

Two approaches are used in modeling of bodies growing during cladding. The first envisages plotting FE-mesh, which covers both the clad part, and all the layers to be deposited in the future. Thus, the number of nodes of FE-mesh does not change during numerical modeling. The essence of the second, more cost-effective approach consists in successive joining of incremental material elements. Here, the number of finite elements changes. The regions of cladding in the first approach or the clad element in the second approach are initially ascribed the properties of «void» material, which is regarded as thermoelastic material with the following characteristics:

$$
E=0 ; \quad v=0,5 ; \alpha=0,
$$

where $E$ is the modulus of elasticity, and $v$ is the Poisson's coefficient.

Value of Poisson's coefficient $v=0.5$ was selected from the condition of correspondence to the properties of molten (incompressible) material, with which the element will be filled. Here, only forming deformation can occur. Thermophysical properties of the «void» are taken to be the same as those of the deposited metal. Therefore, the element is «empty»

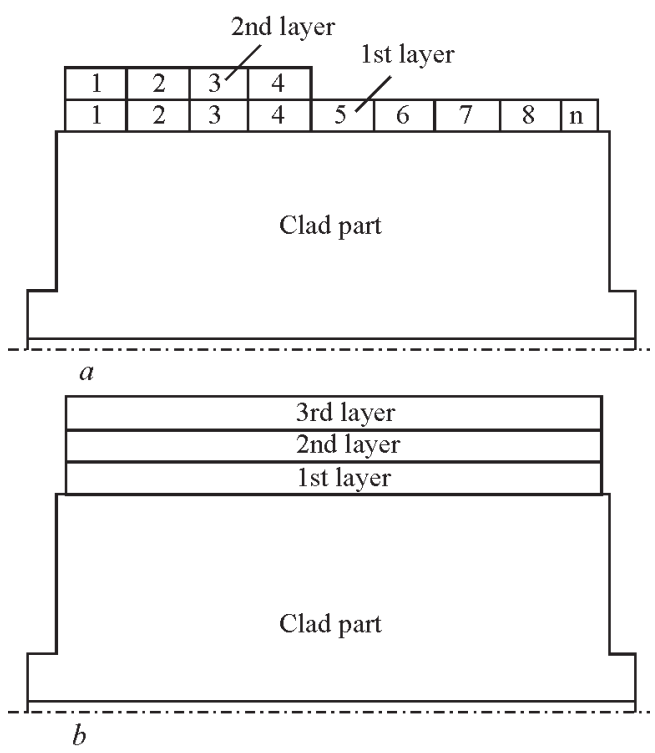

Figure 1. Schematics of models of multilayer cladding: $a-$ bead-by-bead layer-by-layer (figures show bead numbers in the sequence of their deposition in each layer); $b$ - one-time layer-by-layer

only in terms of mechanics. During filling, which is regarded as the process developing in time, «empty» elements of FE-mesh will be filled by material melt being deposited. The calculations allow for the fact that during element filling (cladding) the entire FEmesh is deformed, both the one covering the part, and the «empty» elements.

Let at the moment of filling $t^{*}$, a certain empty element of mesh $\Delta V\left(t^{*}\right)$ adjacent to built-up surface have deformation $\varepsilon_{i j}^{*}$, and let it be filled with material melt of temperature $\theta^{*}$, being non-stressed at the moment of filling. Then, in the clad element

$$
\sigma_{i j}=0, i, j=x, y, z \text { at } t=t^{*} .
$$

Condition (6) in terms of determining equation $\sigma_{i j}=\sigma_{i j}\left(\varepsilon_{k l}, \theta\right)$ can be written as $\sigma_{i j}\left(\varepsilon_{k l}^{*}, \theta^{*}\right)=0$ in mesh element

$$
\Delta V\left(t^{*}\right) i, j, k, l \leftrightarrow x, y, z .
$$

Equality (7) points to absence of stresses (condition (6)) in clad element $\Delta V$, which has initial deformation $\varepsilon_{i j}^{*}$ at filling temperature $\theta^{*}$.

It is assumed that non-elastic deformation of deposited metal is absent at moment of time $t^{*}$ of mesh element filling:

$$
\varepsilon_{i j}^{\mathrm{p}}\left(t^{*}\right)=0 \text { in mesh element } \Delta V\left(t^{*}\right) .
$$

To satisfy conditions (2) and (3), it is necessary at $t>t^{*}$ to modify the equation of Hooke's law for isotropic material (4) as follows:

$$
\begin{gathered}
s_{i j}=2 G_{f}\left(e_{i j}-\varepsilon_{i j}^{\mathrm{p}}-e_{i j}^{*}\right), \\
\sigma_{k k}=2 K_{f}\left(\varepsilon_{k k}-\varepsilon_{k k}^{*}-3 \alpha_{f}\left(\theta-\theta^{*}\right),\right.
\end{gathered}
$$

and to assume in equations (2) and (3): 


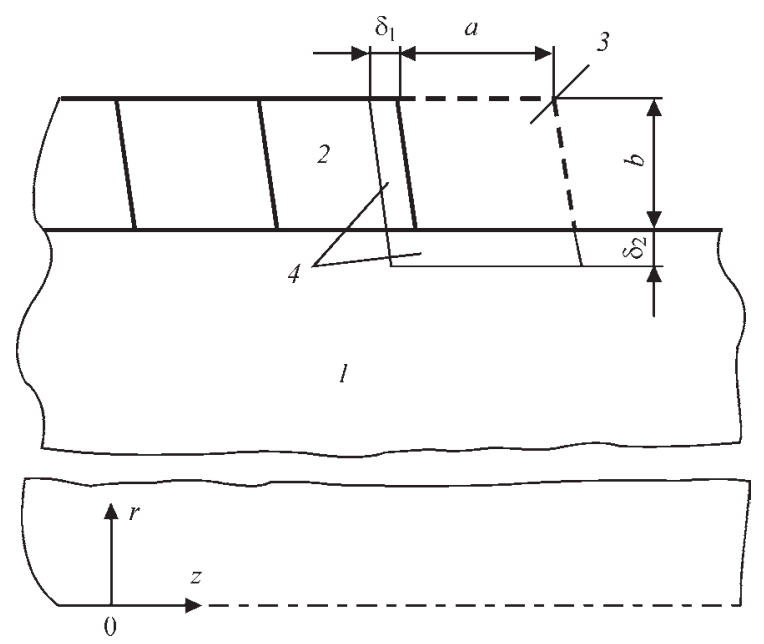

Figure 2. Schematic of filling (cladding) of $N$-th bead: 1 - part; 2 - earlier deposited ( $N-1)$-th bead; 3 - region responsible for next $N$-th bead, which is to be filled (before filling - «empty» region); 4 - regions of subsurface volume heated by welding arc in the part and in earlier deposited bead; $\delta_{1}$ - width of heated region in material of $(N-1)$-th bead during deposition of $N$-th bead; $\delta_{2}$ - width of heated region in base metal during deposition of $N$-th bead (dash-dot line — part axis)

$$
\varepsilon_{i j}^{\mathrm{p}}\left(t^{*}\right)=0, K\left(t^{*}\right)=0, K_{0}\left(t^{*}\right)=K_{0 f}\left(\theta^{*}\right) .
$$

Here, lower index $f$ indicates that the parameters pertain to deposited layer material.

Thus, in order to satisfy condition (6) in subsurface built-up layer, for all elements of clad material determining equations (1)-(4) are individualized by those specific values of strain $\varepsilon_{i j}^{*}$ and temperature $\theta^{*}$, at which their filling occurred at moment of time $t=t^{*}$.

As an example, let us consider spiral cladding of side surface of a cylinder. The schematic of deposited layer filling by individual beads in meridian section (see Figure 1, $a$ ) is given in Figure 2.

Let the start of deposition of $N$-th bead correspond to moment of time $t=t_{N}$. The process is realized in the following sequence:

1. In time interval $\left(t_{N}, t_{N}+t_{Q}\right)$, where $t_{Q}$ is the effective time of source operation, simultaneous heating of the following regions takes places: $(a)$ - «empty» region 3 by uniformly distributed heat source of power $Q_{K}$ (index $K$ means cladding); $(b)$ — adjacent region 4 by welding arc, which is a bulk heat source of power $Q_{L}$ (index $L$ means arc).

2. At moment $t_{N}=t_{N}+t_{Q}$ both the sources switch off and «filling» of region 3 occurs, which is understood to be replacement in this region of «empty» material with (1) properties by material described by system of equations (1)-(4), allowing for modifications (9) and (10), where $\varepsilon_{i j}^{*}$ and $\theta^{*}$ are the strains and temperatures in nodal points of region 3 at the moment of filling.
3. In time interval $\left(t_{N+Q}, t_{N+1}\right)$ cooling takes place due to heat conductivity in-depth of the part and heat exchange with the environment.

4. At moment of time $t=t_{N+1}$ a new mechanically «empty» element is joined and the process is repeated.

Heat coming to the body at cladding is determined by the following relationship:

$$
\bar{Q}=\eta_{T}\left(\eta_{\dot{E}}+\eta_{\dot{Y}}\right) I U \Delta t_{1}=\bar{Q}_{\dot{E}}+\overline{Q_{\dot{Y}}},
$$

where $\eta_{T}$ is the effective power coefficient; $\eta_{\dot{E}}$ is the effective efficiency of part heating by the arc; $\eta_{\dot{Y}}$ is the effective efficiency of heating by electrode metal drops; $\bar{Q}_{\dot{E}}$ and $\bar{Q}_{\dot{Y}}$ are the heats transferred to the part body by the arc and coming to the electrode:

$$
\overline{Q_{\dot{E}}}=\eta_{T} \eta_{\dot{E}} I U \Delta t_{1}, \quad \overline{Q_{\dot{Y}}}=\eta_{T} \eta_{\dot{Y}} I U \Delta t_{1},
$$

where $\Delta t$ is the time of deposition of a number of beads, which is determined, proceeding from geometrical dimensions of clad surface and deposition rate.

Respective bulk powers of heat sources are calculated by the following formulas:

$$
Q_{\dot{E}}=\frac{\overline{Q_{\dot{E}}}}{V_{\dot{E}} t_{Q}}, \quad Q_{\dot{Y}}=\frac{\overline{Q_{\dot{Y}}}}{V_{\dot{Y}} t_{Q}},
$$

where $V_{\dot{E}}$ is the volume of the region, in which the heat source operates, derived by rotation of plane figure 4 or 3 of meridian section about the part axis; $V_{\dot{Y}}$ is the volume of the region derived by rotation of figure 3 (see Figure 2); $t_{Q}$ is the effective time of heat source operation.

The following values of the set of geometrical parameters are assumed for filling schematic (see Figure 2): $\delta_{1}=10^{-3} \mathrm{~m} ; \delta_{2}=0.5 \cdot 10^{-3} \mathrm{~m} ; a$ and $b$ parameters were determined by bead width and height. Values of $\delta_{1}, \delta_{2}$, and $t_{Q}$ were selected using numerical experiment from the condition of approximate equality of temperatures in volumes $V_{\dot{E}}$ and $V_{\dot{Y}}$ at the moment of filling of the region of bead 3 by molten material.

The following values of the given parameters were assumed at determination of heat input [8]: $\eta_{T}=0.6$; $\eta_{\dot{E}}=0.8 ; \eta_{\dot{Y}}=0.2$. Calculations were performed using physico-mechanical characteristics of respective materials. Boundary problem of thermomechanics was solved by FEM. Detailed description of numerical procedure is given in [10-12].

We will give comparison of the results of calculation of residual SSS, derived in terms of schematics of gradual and one-time building-up (see Figure 1).

A cylinder from steel $35 \mathrm{KhM}$ built-up by two layers of $\mathrm{Cr}-\mathrm{Ni}$ steel $10 \mathrm{Kh} 18 \mathrm{~N} 10$ with austenitic-ferritic structure is considered as an example. Cylinder diameter $D=0.126 \mathrm{~m}$, and length is $L=0.25 \mathrm{~m}$. Thickness of the first and second layers is the same and equal 


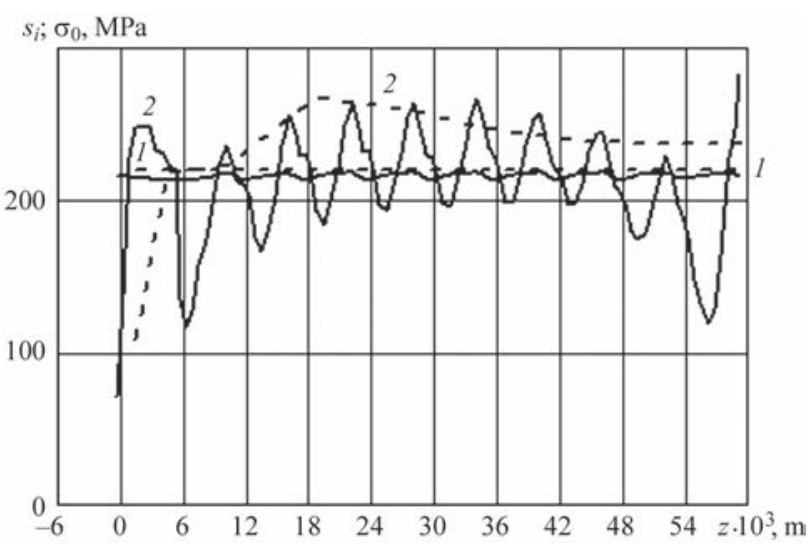

Figure 3. Intensity of stresses $s_{i}(1)$ and mean stress $\sigma_{0}(2)$ in the first deposited layer after deposition of the second layer

to $2.3 \mathrm{~mm}$. Cladding is performed with preheating to $\theta=300{ }^{\circ} \mathrm{C}$.

In the schematic of gradual building-up 10 beads are deposited in each layer to reduce the volume of computations. Calculations showed that the end effects are localized in the zones of the first and the last three beads. Therefore, in the region between the third and seventh beads residual SSS is in place, which can be associated with the region of regular state of the main part of fully built-up cylinder.

Intensity of stress tensor deviator

$$
s_{i}=J_{2}^{1 / 2},
$$

non-elastic deformation intensity

$$
\varepsilon_{i}^{\mathrm{p}}=\left(\frac{1}{2} e_{i j}^{\mathrm{p}} e_{i j}^{\mathrm{p}}\right),
$$

and mean normal stress

$$
\sigma_{0}=\left(\sigma_{r r}+\sigma_{z z}+\sigma_{\varphi \varphi}\right) / 3
$$

are used as SSS characteristics.

Intensity $s_{i}$ is responsible for non-elastic processes and fracture by ductile mode. Mean stress generally identifies tension $\left(\sigma_{0}>0\right)$ or compression state.

Figures 3 and 4 show the distributions of SSS characteristics along $O z$ line at $r=0.064 \mathrm{~m}$ (half of first layer thickness) (here and below solid lines cor-

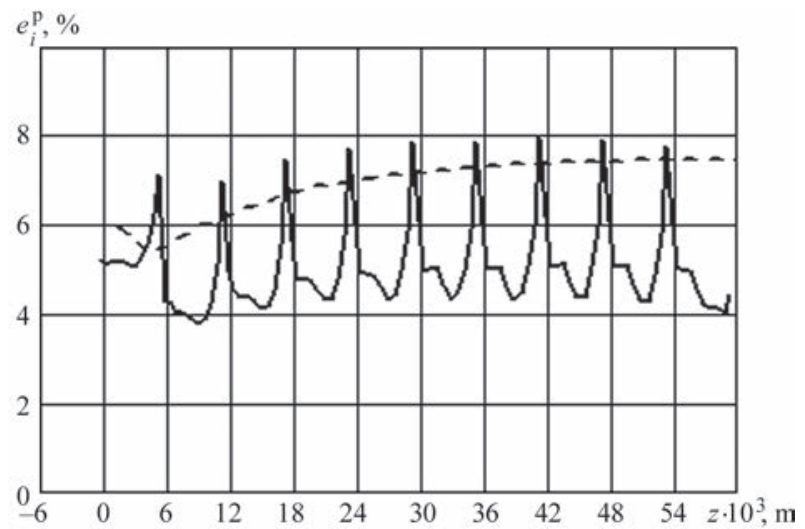

Figure 4. Intensity of residual non-elastic strains in the first deposited layer after second layer deposition

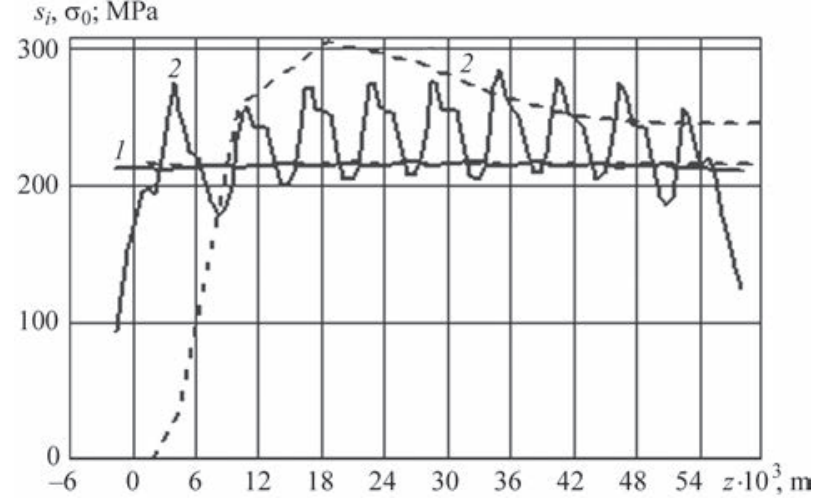

Figure 5. Intensity of stresses $s_{i}(1)$ and mean stress $\sigma_{0}(2)$ in the second deposited layer

respond to the schematic of bead-by-bead deposition, and dash lines indicate one-time layer-by-layer deposition).

Distributions along the median line $r=0.064 \mathrm{~m}$ of residual stresses and strains, which formed in the second built-up layer after its cooling to $20{ }^{\circ} \mathrm{C}$, are represented in Figures 5 and 6.

Analysis of presented results leads to the following conclusions:

1. Schematic of one-time layer-by-layer building-up does not allow for the non-uniform cellular structure of distributions, but describes a smooth averaged change of stress and strain characteristics along the built-up cylinder.

2. Schematic of one-time layer-by-layer building-up corresponds to more extended zones of end disturbances of SSS.

3. In the region of regular distribution of SSS characteristics, calculation results for one-time layer-by-layer schematic are in satisfactory agreement with the results derived on the basis of bead-by-bead deposition schematic. For this reason, the instant layer-by-layer schematic can be used in simplified calculations.

Results of numerical modeling are compared with experimental data. Measurements were performed by hole-drilling method $[13,14]$.

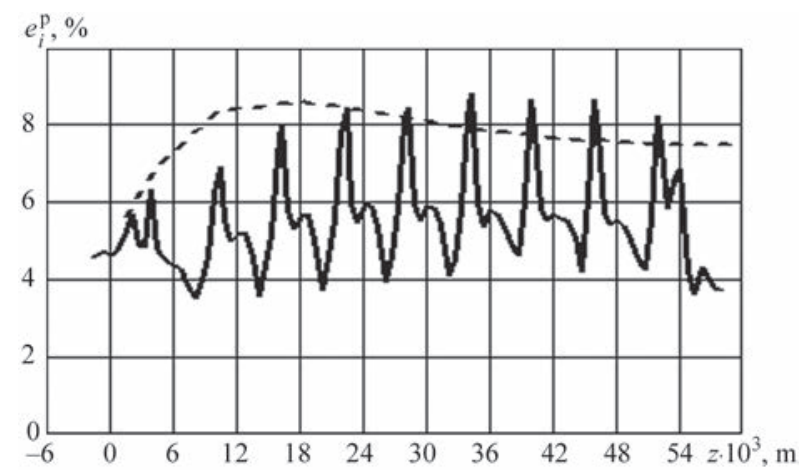

Figure 6. Intensity of residual non-elastic strains in the second deposited layer 


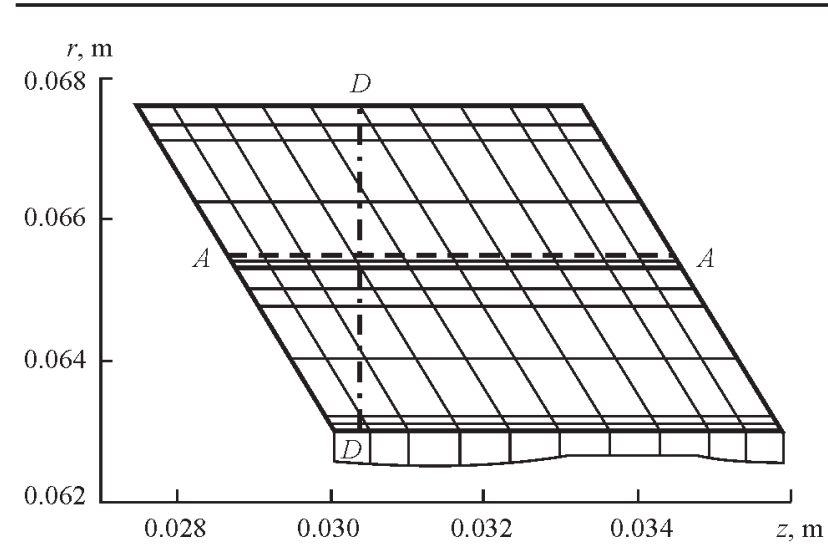

Figure 7. Schematic fragment of deposited layers: dash-dot line $D-D$ - hole axis; dashed line $A-A$ - hole depth; thin lines FE-mesh

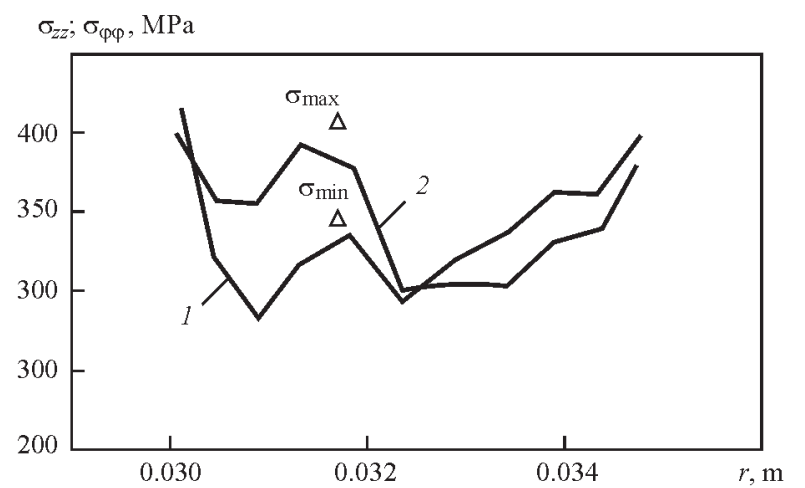

Figure 8. Axial (1) and circumferential (2) stress along line $A-A$ in deposited layer fragment given in Figure 7

Investigations were conducted on sixth bead at two-layer bead-by-bead deposition of layers. Respective fragment of built-up layer is shown in Figure 7. Holes of $1.6 \mathrm{~mm}$ diameter were drilled to depth $\Delta r=2.2 \mathrm{~mm}$ in the center of the bead located in section $z=0.0304 \mathrm{~m}$, to measure the residual stresses.

Figure 8 shows calculated axial distributions of components $\sigma_{z z}$ and $\sigma_{\varphi \varphi}$ in section $A-A$. Triangles indicate experimental values of maximum and minimum principal stresses $\sigma_{\min }$ and $\varphi_{\max }$. It is seen that stress components change quite significantly within the bead. However, good agreement of data is in place in the considered section.

It is more convenient to perform comparison with application of radial stress distributions in built-up layer. According to experimental procedure, measured stresses correspond to and should be compared with the following calculated values in this section:

$$
\begin{aligned}
& \sigma_{\min }=\left\langle\min \left(\sigma_{z z}(r), \sigma_{\varphi \varphi}(r)\right)\right\rangle_{\Delta r}, \\
& \sigma_{\max }=\left\langle\max \left(\sigma_{z z}(r), \sigma_{\varphi \varphi}(r)\right)\right\rangle_{\Delta r} .
\end{aligned}
$$

Here, $\left\langle f(r)>_{\Delta r}\right.$ designates the following value averaged by hole depth:

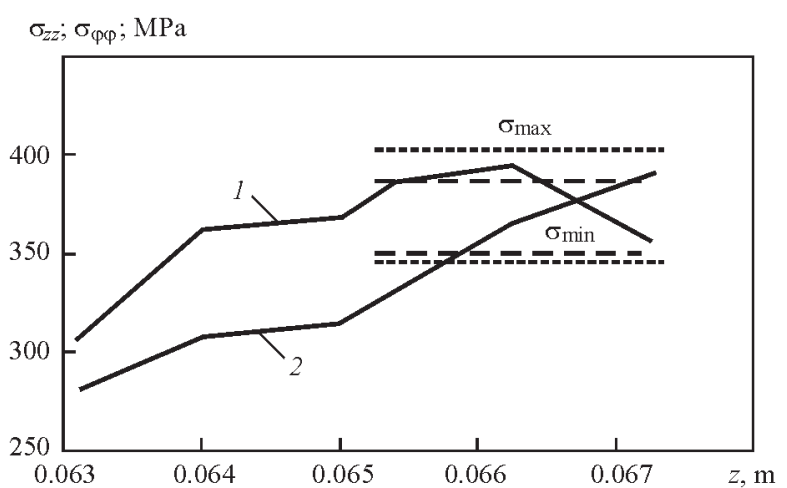

Figure 9. Distribution of residual axial $\sigma_{z z}(1)$ and circumferential $\sigma_{o(}(2)$ stresses across deposited layer thickness in section $z=0.0304 \mathrm{~m}$ : dotted lines - experimental values of principal stresses averaged over thickness in deposited layer; dashed lines - calculated values of these parameters

$$
\langle f(r)\rangle=\frac{2}{r_{0}^{2}-\left(r_{0}-\Delta r\right)^{2}} \int_{r_{0}-\Delta r}^{r_{0}} f(r) r d r,
$$

where $r_{0}$ is the outer radius of built-up bead.

Figure 9 shows the calculated distributions along the radius of components of stresses $\sigma_{z z}$ and $\sigma_{\varphi \varphi}$ in the studied section.

One can see that maximum and minimum principal stresses are not related to any one component. So, component $\sigma_{\varphi \varphi}$ prevails on the surface, and in deeper-lying layers the maximum value is determined by component $\sigma_{z z}$.

\section{Conclusions}

1. Theory of growing bodies and unified model of flow were used as the basis for development of the model of evaluation of thermomechanical state of the part during multilayer cladding, as well as FE procedure of numerical realization of the model. Generally satisfactory agreement of calculated and experimental data is indicative of validity and reliability of the developed approach to modeling the thermomechanical processes in multilayer cladding.

2. In the case of cladding (building-up) a cylinder along its side surface it is found that the schematic of one-time building-up allows quickly enough deriving qualitative evaluation of the level of stresses and strains at analysis of different variants of technological solutions for multilayer cladding of parts. It, however, does not allow for the considerable non-uniform and cellular structure of distributions, but just describes the smooth averaged change of stress and strain characteristics along the cylinder. It does not allow studying such fine technological points as the influence of different schematics of deposited bead overlapping on the part SSS. In this case, it is recommended to apply the procedure of calculation by the schematic of bead-by-bead building-up (deposition). 
1. Balyakin, A.V., Smelov, V.G., Chempinsky, L.A. (2012) Application of additive technologies for development of combustion chamber parts. Vestnik SamaraGAKU, 34(3), 47-51.

2. Marya, S., Hascoet, J.Y., Panigrah, S.K. et al. Additive manufacturing, derivative of welding and joining technology: A literature review. IIW Doc. XII-2185-14; IV-1195-14.

3. Smurov, I.Yu., Konov, S.G., Kotoban, D.V. (2015) About implementation of additive technologies and production in national industry. Novosti Materialovedeniya. Nauka i Tekhnika, $14(2), 11-20$

4. Kovalev, O.B. (2015) Fundamental problems of modeling and diagnostics of processes in laser technologies of additive production of 3D metal pieces. In: Proc. of $11^{\text {th }}$ All-Union Congress on Fundamental Problems of Theoretical and Applied Mechanics (Kazan, Russia, 20-24 Aug. 2015), 1836-1838.

5. Makhnenko, V.I., Kravtsov, T.G. (1976) Thermal processes in mechanized surfacing of parts of circular cylinder type. Kiev: Naukova Dumka.

6. Makhnenko, V.I., Velikoivanenko, E.A., Kravtsov, T.G. et al. (2001) Numerical studies of thermomechanical processes in surfacing of shafts of ship mechanisms. The Paton Welding $J ., 1,2-10$.
7. Arutyunyan, N.Kh., Drozdov, A.D., Naumov, V.E. (1987) Mechanics of growing of viscous-elastic-plastic bodies. Moscow: Nauka.

8. (2006) Technology of welding, brazing and cutting: Encyclopedia, Vol. III-4. Ed. by B.E. Paton. Moscow: Mashinostroenie.

9. Bodner, S.R. (2000) Unified plasticity - an engineering approach: Final report. Haifa: Israel IT.

10. Ryabtsev, I.A., Senchenkov, I.K. (2013) Theory and practice of surfacing operations. Kiev: Ekotekhnologiya.

11. Ryabtsev, I.A., Senchenkov, I.K., Turyk, E. (2015) Surfacing, materials, technologies, mathematical modeling. Gliwice: Silesia PI.

12. Senchenkov, I.K., Ryabtsev, I.A., Turyk, E. et al. (2005) Calculation of residual stresses in multilayer helical cladding of cylindrical parts on the base of theory of growing of viscous-plastic bodies. Svarochn. Proizvodstvo, 9, 18-25.

13. ASTM Standard E 837: Standard test method for determining residual stresses by the hole drilling strain-gage method.

14. (1993) Measurement of residual stresses by the hole drilling strain gage method. Measur. Group Inc. Tech. Note.

Received 05.04.2016

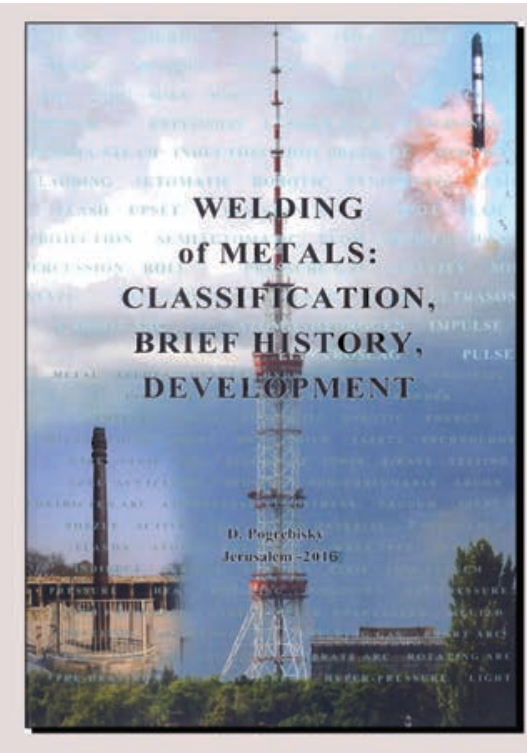

NEWW BOOK

Pogrebisky D. (2016) Welding of Metals: Classification, Brief History, Development, 380 pp., 250 pictures, a dozen tables, $205 \times 290 \mathrm{~mm}$, soft cover, Jerusalem, Israel.

The book price is $\$ 175$.

In June, 2016 the former collaborator of Paton Welding Institute published in Jerusalem a new book.

The book «Welding of Metals: Classification, Brief History, Development» gives a lot of information about metals welding processes, including not listed in American and European normative documents which author tries to lead to the complete match to each other. With the aim of international harmonization, a preliminary attempt was made to produce a unified classification of about 300 welding methods, basing on the abbreviations that are similar to adopted ones of the American Welding Society.

At the same time the book author tried to build the impartial review of historical metals welding development against a background of general sciences and adjacent processes progress. Some comparative, economic and other considerations are given too - together with a rich illustrative material and large references.

The book may be useful for different welding specialists, industrial engineers, designers of precision metal structures and various electro-mechanical devices as well as for students of relevant specialties.

The main purpose of the book is to contribute to general picture of welding development. There are mentioned much more than 300 welding methods that were developed in the former Russia, USSR, in USA, England, Germany, France, Sweden, Japan, Finland, Austria, China, Australia, Brazil, Switzerland and many other countries.

\author{
Orders for book please send to: \\ POB 31445, Jerusalem 91313, Israel \\ E-mail: davidpogrebis@gmail.com \\ www.actualweld.com
}

